\title{
Carrier Frequency Offset Estimation for Uplink OFDMA using Partial FFT Demodulation
}

\author{
Srinivas Yerramalli, Milica Stojanovic and Urbashi Mitra
}

\begin{abstract}
Fast and accurate Carrier Frequency Offset (CFO) estimation is a problem of significance in many multi-carrier modulation based systems, especially in uplink Orthogonal Frequency Division Multiple Access (OFDMA) where the presence of multiple users exacerbates the inter-carrier interference (ICI) and results in multi-user interference (MUI). In this paper, a new technique called partial FFT demodulation is proposed. Estimators for the CFO are derived by considering an approximated matched filter for each user, implemented efficiently using several FFTs operating on sub-intervals of an OFDM block. Through simulations, we demonstrate the feasibility and performance of the derived estimators, and discuss the associated trade-offs.
\end{abstract}

\section{INTRODUCTION}

Orthogonal Frequency Division Multiplexing (OFDM) has emerged as the primary signaling scheme for high speed digital communication systems such as WiMax, WiFi, $4^{\text {th }}$ generation mobile systems and is under consideration for underwater acoustic communications. Interest in OFDM stems from the fact that it can decompose a frequency selective channel into several independent flat channels enabling single tap equalization and symbol-by-symbol detection. However, OFDM is sensitive to carrier frequency offset (CFO) and time-varying channels which introduce inter-carrier interference. Furthermore, for uplink Orthogonal Frequency Division Multiple Access (OFDMA), CFOs for different users result in self-ICI and multi-user interference (MUI).

In this paper, we focus on CFO estimation for OFDM and uplink OFDMA. Subcarrier assignment schemes, which influence the design of CFO compensation in uplink OFDMA, can be broadly classified into four categories [1], [4]. In the subband Carrier Assignment Scheme (SCAS), a large block of subcarriers is allocated to one user while in an Interleaved Carrier Assignment Scheme (ICAS), subcarriers are allocated periodically to each user. In the Generalized Carrier Assignment Scheme (GCAS), flexible dynamic subcarrier allocation occurs. Finally, in standardized OFDMA uplink systems such as 802.16e [1], a new Tile (group of adjacent subcarriers) based Subcarrier Assignment Scheme (TCAS) which combines the simplicity of SCAS and retains the flexibility of GCAS systems is used.

The problem of CFO estimation for uplink OFDMA has been of considerable interest. Filter-banks separate different users in [9], enabling per user CFO estimation and is suitable for SCAS. Energy minimization of null subcarriers is

This research has been funded in part by the following grants and organizations: ONR MURI grant N00014-07-1-0738, ONR N00014-09-10700, ONR N00014-10-1-0576, NSF 0831728, NSF CNS-0832186, NSF CNS-0821750 (MRI), NSF OCE-0520324 and the University of Southern California's Provost Office. employed in [2]; while in [3] the periodic structure of ICAS is exploited for CFO estimation. In [11], a blind estimator is proposed based on signal kurtosis. For GCAS OFDMA, a maximum likelihood (ML) solution based on an exhaustive multi-dimensional search was proposed in [6]. An alternating projection frequency estimator (APFE) approximated the multi-dimensional search via iterating over single dimensions, thus reducing complexity. An approximate version of the APFE (called AAPFE) which replaces the inversion of a CFOdependent matrix in APFE with a pre-determined approximate matrix is proposed in [7]. Several algorithms which aim to reduce computational complexity by using some constraints on signaling or iterative search have also been proposed for GCAS systems (see DUFE estimator [10], line search [5], etc.). In [8], a training sequence structure which can be realized by tile-based systems is proposed to estimate the CFO of different users.

In this paper, we investigate CFO estimation for TCAS and SCAS uplink OFDMA systems. We introduce the idea of partial FFT demodulation, where the outputs of several partial FFTs are used instead of a full FFT at the receiver. Using partial FFT demodulation, we show that the CFO can be approximately modeled as a progressively increasing phase distortion and derive an estimation algorithm for the CFO in OFDM systems. Applying this approach to uplink OFDMA systems allows us to approximately decouple the CFO estimation problem for each user in TCAS and SCAS systems. The partial FFT approach does not impose any constraints on the training sequences, utilizes only one OFDMA symbol and offers a significant reduction in computational cost as all the quantities required can be pre-computed based on the knowledge of subcarrier allocation for each user. Numerical simulations demonstrate the effectiveness of this approach.

The paper is organized as follows. Section II describes the signal model for uplink OFDMA, introduces the idea of partial FFT demodulation and demonstrates the estimation of CFO for a single user OFDM system. Section III applies the partial FFT approach to uplink OFDMA systems and evaluates the performance of this algorithm under several conditions. Numerical results are presented in Section IV. Section V concludes the paper.

\section{Signal Model And Partial FFT Demodulation}

\section{A. OFDMA Signal Model}

We consider an uplink OFDMA system with $K$ subcarriers and $N_{u}$ users. The subcarriers are divided among the users using a predetermined optimization criterion. The base station 
is assumed to know the subcarrier allocation for each active user. Let $\mathcal{K}_{n}$ be the set of subcarriers allocated to the $n^{\text {th }}$ user and $\mathcal{K}_{n 1} \cap \mathcal{K}_{n 2}=\phi$. At each mobile station, all the OFDM subcarriers allocated to the $n^{t h}$ user are modulated using independent and identically distributed information symbols drawn from a finite constellation. Let $T$ and $T_{g}$ denote the duration of the OFDM symbol and the cyclic prefix (CP) respectively. The first subcarrier is at a frequency $f_{0}$, and the subcarrier spacing is $\Delta f=1 / T$. The $k^{t h}$ subcarrier frequency for all users is $f_{k}=f_{0}+(k-1) \Delta f$, and the total signaling bandwidth is $B=K \Delta f$. In this paper, we assume that the cyclic prefix is longer than the maximum delay spread of all the users and that this allows perfect timing synchronization. The passband OFDM transmitted signal for the $n^{t h}$ user is then

$$
s^{(n)}(t)=\sum_{l \in \mathcal{K}_{n}} d_{l} e^{j 2 \pi f_{l} t}, t \in\left[-T_{g}, T\right] .
$$

We consider a quasi-static fading channel where the channel between each user and the base station is assumed to be stationary for one OFDMA signalling interval duration but can change from one interval to another. The channel from the $n^{t h}$ user to the base station can be modeled as

$$
h^{(n)}(\tau)=\sum_{p=1}^{P_{n}} h_{p}^{(n)} \delta\left(\tau-\tau_{p}^{(n)}\right)
$$

where $h_{p}^{(n)}$ and $\tau_{p}^{(n)}$ are the channels gain and the path delays for the $p^{t h}$ path on the $n^{t h}$ uplink. The signal from each user travels through a different channel. Let us assume that the $n^{\text {th }}$ link has a CFO of $f_{d}^{(n)}$. The signal at the base station arriving from the $n^{t h}$ user, after removing the CP, can then be expressed as

$$
\begin{aligned}
r^{(n)}(t) & =\sum_{p=1}^{P_{n}} h_{p}^{(n)} s\left(t-\tau_{p}^{(n)}\right) e^{j 2 \pi f_{d}^{(n)} t}, t \in\left[-T_{g}, T\right] \\
& =\sum_{l \in \mathcal{K}_{n}} d_{l} H_{l}^{(n)} e^{j 2 \pi\left(f_{l}+f_{d}^{(n)}\right) t},
\end{aligned}
$$

where $H_{l}^{(n)}=\sum_{p=1}^{P_{n}} h_{p}^{(n)} e^{-j 2 \pi f_{l} \tau_{p}^{(n)}}$ is the channel frequency response on the $l^{t h}$ subcarrier for the $n^{t h}$ user. The signal at the base station is the sum of contributions from all the active users and is given as

$$
r(t)=\sum_{n=1}^{N_{u}} \sum_{l \in \mathcal{K}_{n}} d_{l} H_{l}^{(n)} e^{j 2 \pi\left(f_{l}+f_{d}^{(n)}\right) t}+w(t),
$$

where $w(t)$ is additive white Gaussian noise with power spectral density $N_{0}$.

\section{B. Partial FFT Demodulation}

To illustrate the idea of partial FFT demodulation, we first consider a conventional OFDM system with no carrier frequency offset. The received signal can then be simplified as

$$
r(t)=\sum_{l} d_{l} H_{l} e^{j 2 \pi f_{l} t}+w(t)
$$

where $H_{l}$ is the channel frequency response on the $l^{\text {th }}$ subcarrier. For simplicity of notation, we drop the user index $n$ for the current scenario. For conventional OFDM demodulation, the output of the $k^{\text {th }}$ demodulator, operating on the interval $[0, T]$, is given as

$$
r_{k}=\frac{1}{T} \int_{0}^{T} r(t) e^{-j 2 \pi f_{k} t} d t, k=1,2, \cdots, K .
$$

In partial FFT demodulation, the useful OFDM symbol duration $T$ is divided into $M$ non-overlapping intervals and each interval is assigned to a demodulator which performs an integration on the partial interval $[(m-1) T / M, m T / M], m=$ $1,2, \cdots, M$. The output of the $m^{\text {th }}$ partial FFT demodulator for the $k^{t h}$ subcarrier can then be expressed as

$$
\begin{aligned}
r_{k}(m) & =\frac{1}{T} \int_{\frac{(m-1) T}{M}}^{\frac{m T}{M}} r(t) e^{-j 2 \pi f_{k} t} d t \\
& =\sum_{l} d_{l} H_{l} I_{l-k}(m)+w_{k}(m),
\end{aligned}
$$

where $w_{k}(m)$ is the zero mean additive white Gaussian noise at the output of the $m^{t h}$ demodulator. The noise $w_{k}(m)$ is independent for different $m$ for a fixed subcarrier, but is correlated for a fixed $m$ across subcarriers. The function $I_{i}(m)$ describes the effect of partial integration:

$I_{i}(m)=\frac{1}{T} \int_{\frac{(m-1) T}{M}}^{\frac{m T}{M}} e^{j 2 \pi i \Delta f t} d t, i=-(K-1), \cdots,(K-1)$.

This function $I_{i}(m)$ obeys a key property which is the motivation for partial FFT demodulation:

$$
\sum_{m=1}^{M} I_{i}(m)=\delta_{i}
$$

For a time-invariant channel, the outputs of the partial FFT demodulators can be summed directly and the resulting value will be the same as that obtained taking a full interval FFT of the received signal, i.e., $\sum_{m} r_{k}(m)=r_{k}$.

\section{Partial FFT outputs in the presence of $C F O$}

We now investigate the structure of the partial FFT outputs in the presence of a frequency offset $f_{d}$. The received signal in the presence of CFO can be modeled as

$$
r(t)=\sum_{l=1}^{K} d_{l} H_{l} e^{j 2 \pi f_{l} t} e^{j 2 \pi f_{d} t}+w(t)
$$

The output of the $m^{\text {th }}$ partial FFT demodulator for the $k^{t h}$ OFDM subcarrier is then

$y_{k}(m)=\frac{1}{T} \sum_{l=1}^{K} d_{l} H_{l} \int_{\frac{(m-1) T}{M}}^{\frac{m T}{M}} e^{j 2 \pi\left(f_{l}-f_{k}\right) t} e^{j 2 \pi f_{d} t} d t+w_{k}(m)$,

where $w_{k}(m)$ is additive noise. Let us define $\epsilon_{0}=f_{d} T$ as the normalized CFO for this link. Noting that the rate of change of $e^{j 2 \pi f_{d} t}$ is much slower than the rate of change of $e^{j 2 \pi\left(f_{l}-f_{k}\right) t}$, the output of the integral in (12) can be approximated using a midpoint value for $e^{j 2 \pi f_{d} t} d t$. The approximated partial FFT 

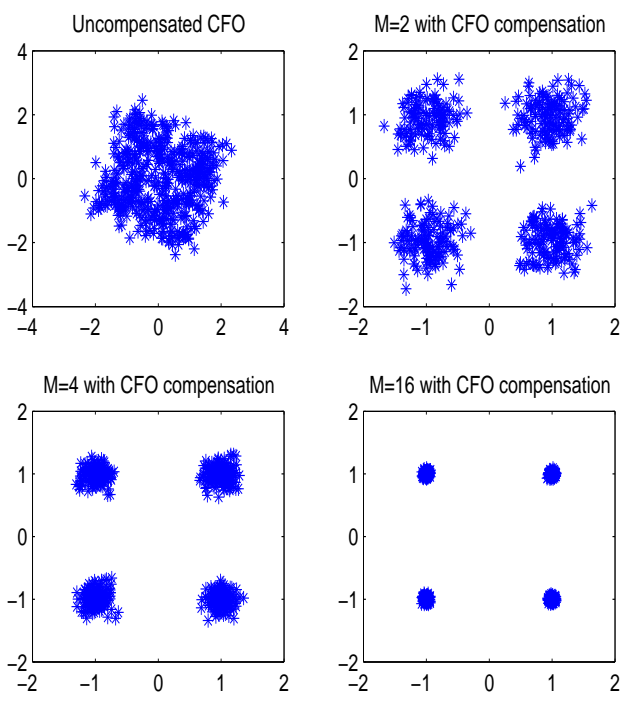

Fig. 1: The improvement obtained by pre-compensating the phase distortion and combining the partial FFT outputs for a one tap, unit gain and noiseless receiver for a 4-QAM constellation.

outputs can be modeled as

$$
y_{k}(m) \approx e^{j 2 \pi \epsilon_{0} \frac{2 m-1}{2 M}} \sum_{l=1}^{K} d_{l} H_{l} I_{l-k}(m)+w_{k}(m),
$$

We note that the accuracy of approximating $y_{k}(m)$ is better for moderate to large values of $M$. By pre-compensating the partial FFT outputs before combining, according to $\sum_{m=1}^{M} e^{-j 2 \pi \epsilon_{0} \frac{2 m-1}{2 M}} y_{k}(m) \approx d_{k} H_{k}$, the distortion due to CFO can be reduced significantly as $M$ increases. In practice, partial FFT demodulation can be implemented efficiently using several FFT blocks whose inputs are appropriately zero padded.

Fig. 1 shows the scatter plots of the received 4-QAM symbols transmitted through a one-tap, unit-gain channel and a noiseless receiver when $\epsilon_{0}=0.3$. Shown in the top left corner is the conventional FFT output derived from (12). We see that the constellation is phase rotated and suffers from significant ICI as well. The other three plots show the scatter plot of the received symbols when they are pre-compensated for the phase distortion $e^{j 2 \pi \epsilon_{0} \frac{2 m-1}{2 M}}$ and then added together for $M=2,4,16$. The phase rotation is clearly eliminated in the constellation and the ICI is significantly reduced.

\section{CFO Estimation From Partial FFT Outputs}

By now, we have shown that pre-compensating for the distortion effects before combining can significantly reduce the ICI. However, in doing so we have assumed accurate knowledge of the normalized CFO $\epsilon_{0}$. We now show that a good estimate of the CFO can be obtained from the partial FFT outputs $y_{k}(m)$ themselves. For simplicity of illustration, we consider an OFDM symbol with all the subcarriers used as pilots. This can easily be generalized to the scenario where an OFDM symbol carriers both pilot symbols and the data.
Let us define $\mathbf{D}=\operatorname{diag}\left[d_{1}, d_{2}, \cdots, d_{K}\right]$ as the diagonal matrix containing the transmitted information symbols, and let $\mathbf{r}(m)=\left[r_{1}(m), r_{2}(m), \cdots, r_{K}(m)\right], m=1,2, \cdots, M$ be the outputs of the $m^{\text {th }}$ partial FFT demodulator in vector form. We propose a maximum likelihood estimator (MLE) to estimate the value of the CFO. For a candidate value of $\epsilon$, we first combine the partial FFT outputs as

$$
\mathbf{y}_{\epsilon}=\sum_{m=1}^{M} e^{-j 2 \pi \epsilon \frac{2 m-1}{2 M}} \mathbf{r}(m)
$$

Assuming that the correct value of $\epsilon$ is used and that $\mathbf{r}$ is free of distortion, we can express the combined output $\mathbf{y}_{\epsilon}$ in terms of the transmitted symbols and the channel impulse response as $\mathbf{y}_{\epsilon}=\mathbf{D F h}+\mathbf{v}$, where, $\mathbf{F}$ is the matrix containing the first $P$ columns of the DFT matrix, and $\mathbf{h}=\left[h_{1}, h_{1}, \cdots, h_{P}\right]$ is the vector of channel impulse response coefficients. The estimated channel impulse response for the candidate value $\epsilon$ is given as $\hat{\mathbf{h}}_{\epsilon}=\mathbf{F}^{\dagger} \mathbf{D}^{-1} \mathbf{y}_{\epsilon}$. Treating the channel impulse response coefficients as nuisance parameters and substituting back their ML estimate in $\mathbf{y}_{\epsilon}$, the ML estimator for the CFO can be derived as

$$
\hat{\epsilon}_{0}=\arg \min _{\epsilon}\left|\mathbf{y}_{\epsilon}-\mathbf{D F F} \mathbf{D}^{\dagger} \mathbf{D}^{-1} \mathbf{y}_{\epsilon}\right|^{2}
$$

This estimator exploits the fact that in the absence of CFO, the weighted combination of the partial FFT outputs is confined to a subspace spanned by the columns of $\mathbf{F}$. Hence, for identifiability of $\epsilon$, the number of pilot symbols used for estimation (length of $\mathbf{y}_{\epsilon}$ ) should be at least one more than the length $P$ of the impulse response.

Weighted combining of the partial FFT outputs effectively acts as front-end filtering and it requires only a minor increase in computational complexity (several additional FFTs). When the distortion of the received signal is due to CFO, combining the de-rotated partial FFT outputs can be interpreted as an approximate implementation of the matched filter for each subcarrier. We note that although partial FFT combining reduces ICI significantly, it does not fully eliminate it as seen from Fig. 1. For a discrete implementation, ICI is completely eliminated only when $M=K$. The de-rotation of the outputs (see (13)) by the correct value of CFO not only corrects the distortion due to $\mathrm{CFO}$ on the desired carrier but also reduces the interference from adjacent carriers. This behavior can be exploited to estimate the CFOs of multiple users in uplink OFDMA systems in which each user is allocated a large group of adjacent subcarriers (subband CAS) or several smaller groups of subcarriers (tiled CAS as proposed in the OFDMA standard [1]).

\section{UPLINK OFDMA CARRIER FREQUENCY ESTIMATION}

In this section, we apply the partial FFT demodulation method to estimate the CFOs of several users in uplink OFDMA systems. We consider SCAS and TCAS uplink OFDMA, where several subcarriers of one user are located adjacent to each other. The received signal at the base station 
for the uplink is then (from (5))

$$
r(t)=\sum_{n=1}^{N_{u}} \sum_{l \in \mathcal{K}_{n}} d_{l} H_{l}^{(n)} e^{j 2 \pi\left(f_{l}+f_{d}^{(n)}\right) t}+w(t),
$$

As described in the conventional OFDM scenario in Section II, the received signal $r(t)$ is input to several partial interval FFT blocks. The output of the $m^{\text {th }}$ block is given as

$$
\begin{aligned}
& y_{k}(m)=\frac{1}{T} \int_{\frac{(m-1) T}{M}}^{\frac{m T}{M}} r(t) e^{-j 2 \pi f_{k} t} d t, m=1,2, \cdots, M \\
= & \frac{1}{T} \sum_{n} \sum_{l \in \mathcal{K}_{n}} d_{l} H_{l}^{(n)} \int_{\frac{(m-1) T}{M}}^{\frac{m T}{M}} e^{j 2 \pi\left(f_{l}-f_{k}+f_{d}^{(n)}\right) t}+w_{k}(m),
\end{aligned}
$$

where $w_{k}(m)$ is the additive Gaussian noise. Let us define $\epsilon^{(n)}=f_{d}^{(n)} T$ as the normalized CFO for the $n^{t h}$ user. As in Section II, the output of the $m^{t h}$ partial FFT demodulator for the $k^{t h}$ subcarriuer can be approximated as

$$
y_{k}(m) \approx \sum_{n=1}^{N_{u}} \sum_{l \in \mathcal{K}_{n}} e^{j 2 \pi \epsilon^{(n) \frac{2 m-1}{2 M}}} d_{l} H_{l}^{(n)} I_{l-k}(m)+w_{k}(m) .
$$

In an OFDM system with receiver impairments, we note that most of the ICI is due to the subcarriers adjacent to the one of interest. When a group of contiguous subcarriers are allocated to one user, we note that for the subcarriers in the middle of the group mostly experience self ICI, while subcarriers at the edge of the group experience both ICI and MUI. For the subcarriers which do not border any group, when the phase distortion is properly compensated, the ICI and the MUI can be neglected to simplify the estimation problem. The partial FFT outputs can then be further approximated as

$y_{k}(m) \approx \sum_{l \in \mathcal{K}_{n}} e^{j 2 \pi \epsilon^{(n)} \frac{2 m-1}{2 M}} d_{l} H_{l}^{(n)} I_{l-k}(m)+w_{k}(m), k \in \mathcal{K}_{n}$.

We emphasize again that the second approximation does not hold true in general for subcarriers whose adjacent carriers belong to different users, i.e., the edges of subbands and tiles for subband CAS and tiled CAS, respectively.

Ignoring the MUI allows us to decouple the CFO estimation problem for each user and reduces the multi-parameter joint estimation problem into a series of single parameter estimation problems for each user. For the $n^{t h}$ user and a candidate value of $\mathrm{CFO} \epsilon$, let us define $\mathbf{y}_{\epsilon}^{(n)}$ as the vector of phase compensated and combined data:

$$
\mathbf{y}_{\epsilon}^{(n)} \approx \mathbf{D}^{(n)} \mathbf{F}^{(n)} \mathbf{h}^{(n)}+\mathbf{w}^{(n)}
$$

As in the OFDM scenario, treating the channel impulse response coefficients as nuisance parameters and replacing them by their estimates, the estimate of the CFO is given as

$$
\hat{\epsilon}^{(n)}=\arg \min _{\epsilon}\left|\mathbf{y}_{\epsilon}^{(n)}-\mathbf{D}^{(n)} \mathbf{F}^{(n)}\left(\mathbf{F}^{(n)}\right)^{\dagger}\left(\mathbf{D}^{(n)}\right)^{-1} \mathbf{y}_{\epsilon}^{(n)}\right|^{2},
$$

where $\mathbf{D}^{(n)}$ is the diagonal matrix of pilot symbols modulated on all the carriers allocated to the $n^{\text {th }}$ user and $\mathbf{F}^{(n)}=$ $\left[e^{j \frac{2 \pi}{K} a b}\right], a \in \mathcal{K}_{n}, b=0,1, \cdots, P-1$.

\section{A. Tradeoffs in Estimation Performance}

The estimation error of the proposed algorithm depends on the number of partial FFT segments $M$, the signal to noise ratio (SNR) and the CFO spread $\eta$ among the users which we define as $\eta=\max \left|\epsilon^{(r)}-\epsilon^{(s)}\right|, r, s \in\left[1,2, \cdots, N_{u}\right]$. For a single-user OFDM system, as the value of $M$ increases, the accuracy of approximating the CFO in (13) increases and the residual ICI due to approximation error reduces. This results in better estimation performance, especially at high SNR, where the effect of residual ICI dominates over the noise. A larger value of $M$ requires a few additional partial interval FFTs, which slightly increases the computational complexity.

For multi-user uplink OFDMA, the CFO spread $\eta$ is the key parameter which influences the estimation accuracy. For smaller $\eta$, i.e. when the $\mathrm{CFO}$ values for each user are close to each other, the estimation accuracy should be close to that of a single-user scenario as the MUI is negligible. However, for large values of $\eta$, we expect an error floor for high SNR as MUI is ignored in deriving the estimator. The size of each tile in TCAS allocation also plays an important role in determining the performance of the system. For larger tile sizes, the estimation error should approach the single user scenario as the MUI is greatly reduced in this case.

\section{Simulation Results and Discussion}

In this section, we present simulation results to demonstrate the performance of the proposed CFO estimation algorithms using partial FFT demodulation. We consider an OFDMA based WiMax system with $N_{u}$ users and $K=512$ subcarriers. The subcarrier spacing is $\Delta f=39 \mathrm{kHz}$ and the OFDMA symbol duration is $T=25.6 \mu \mathrm{s}$. The total bandwidth occupied by the system is $B=K \Delta f=20 \mathrm{MHz}$. The center frequency is assumed to be $f_{c}=5 \mathrm{GHz}$. For the uplink of each user, we consider a 6 tap channel with the path delays chosen at random and a maximum delay spread of $2 \mu \mathrm{s}$. To eliminate inter-block interference from previous OFDMA symbols, the cyclic prefix is assumed to be of length $T_{g}=T / 8=3.2 \mu \mathrm{s}$. For each active link, the normalized CFO [6] is considered to range within $[-0.3,0.3]$. We assume that the system supports a maximum of 8 users with 64 subcarriers allocated to each user. When SCAS allocation is used for the uplink, it is assumed that a block of 64 subcarriers are allocated sequentially to each user and there is MUI at the edge subcarriers for each user. For TCAS allocation, the 64 carriers of each user are divided into 8 groups of 8 subcarriers each, and these groups are allocated randomly.

Fig. 2 shows the CFO estimation error as a function of SNR for TCAS and SCAS allocation for various values of $M$ for a single-user scenario. As $M$ increases, the performance improvement at low and moderate SNRs is modest, but considerable improvement is observed in the high SNR regime. This is due to the fact that as $M$ increases, the power of the residual ICI reduces and this considerably improves the performance when ICI dominates over the noise.

Figs. 3 and 4 show the average frequency estimation error (averaged over all the users) for SCAS and TCAS allocation, respectively, for $N_{u}=1,3,6$ and $M=16$ as a function of 


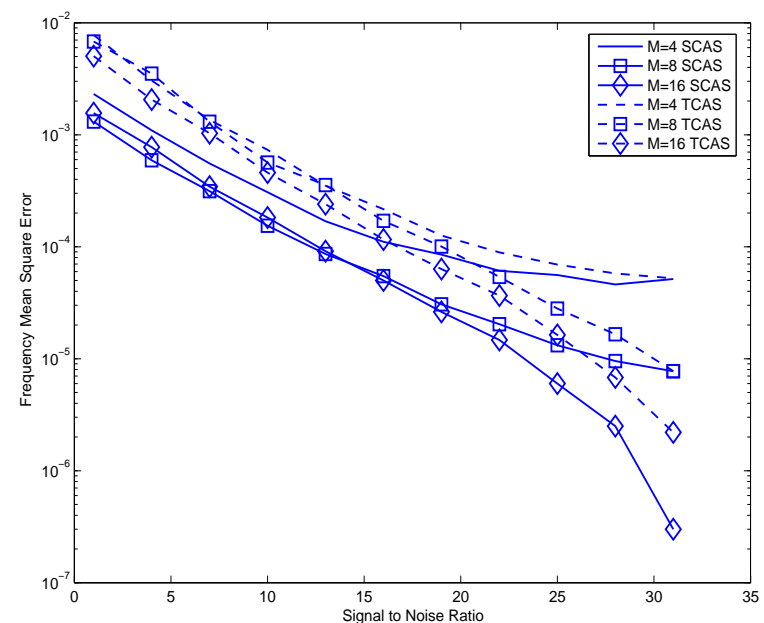

Fig. 2: CFO estimation error for one user OFDMA system with TCAS and SCAS allocation for varying values of $M$ as a function of SNR

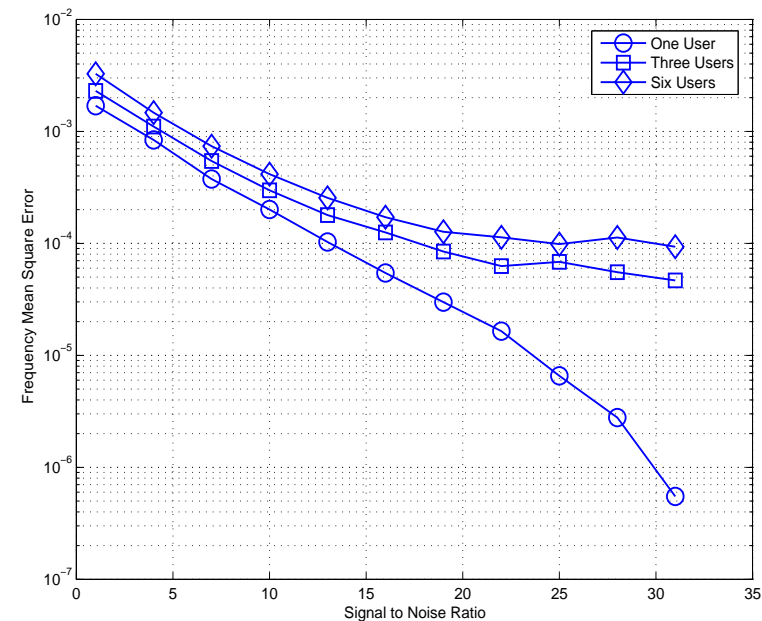

Fig. 3: CFO estimation error for SCAS allocation for varying number of users as a function of SNR, $M=16$

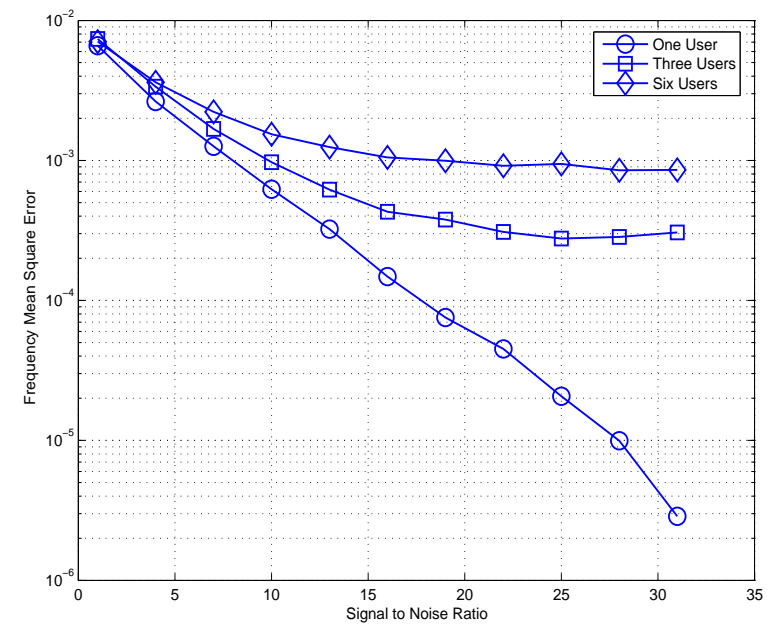

Fig. 4: CFO estimation error for TCAS allocation for varying number of users as a function of SNR, $\mathrm{M}=16$
SNR. We note that all the subcarriers allocated to the users (including the edge subcarriers which suffer from extreme MUI) are used for CFO estimation. It is observed that in the presence of multiple users, the estimation error exhibits a floor. This can be attributed to the fact that the algorithm has been derived by ignoring the MUI from other users.

\section{Conclusions}

CFO estimation for uplink OFDMA systems is affected by the presence of multiple users who share the available subcarriers. This results not only in ICI, but also in MUI at the uplink base station. In this paper, a new technique called partial FFT demodulation has been introduced in which FFT processing is performed over partial segments of the OFDM symbol, such that each segment can be weighed before combining. Exploiting the structure in the resulting signal, enables a simple low-complexity estimator for the CFO. The performance of partial FFT demodulation/CFO estimation was investigated in light of SCAS and TCAS uplink OFDMA systems, showing a good estimation performance at very low complexity.

\section{REFERENCES}

[1] "IEEE standard for Local Area Metropolitan Networks, Part 16: Air interface for Fixed and Mobile Broadband Wireless Access Systems Amendment 2: Physical and Medium Access Control Layers for Combined Fixed and Mobile Operation in Licensed Bands, IEEE Std. $802.16 \mathrm{e}, " 2008$.

[2] S. Barbarossa, M. Pompili, and G. B. Giannakis, "Channel-independent synchronization of Orthogonal Frequency Division Multiple Access systems," IEEE Journal of Selected Areas in Communications, vol. 20, no. 2, Feb 2002.

[3] Z. Cao, U. Tureli, and Y.-D. Yao, "Deterministic multiuser carrierfrequency offset estimation for interleaved OFDMA uplink," IEEE Transactions on Communications, vol. 52, no. 2, Feb 2004.

[4] M. Morelli, C. C. J. Kuo, and M. O. Pun, "Synchronization techniques for Orthogonal Frequency Division Multiple Access (OFDMA): A Tutorial Review," Proceedings of the IEEE, vol. 95, no. 7, pp. 13941427, Aug 2007.

[5] Y. Na and H. Minn, "Line search based iterative joint estimation of channels and frequency offsets for uplink ofdma systems," IEEE Transactions on Wireless Communications, vol. 6, no. 12, Dec 2007.

[6] M. on Pun, M. Morelli, and C. C. J. Kuo, "Maximum likelihood synchronization and channel estimation for OFDMA uplink transmissions," IEEE Transactions on Communications, vol. 54, no. 4, Apr 2006.

[7] S. Sezginer and P. Bianchi, "Asymptotically efficient reduced complexity frequency offset and channel estimators for uplink MIMO-OFDMA systems," IEEE Transactions on Signal Processing, vol. 56, no. 3, Mar 2008.

[8] P. Sun and L. Zhang, "A simple iterative carrier frequency offset synchronization technique for ofdma uplink transmissions," Wiley Interscience Journal on Wireless Communications and Mobile Computing, 2010.

[9] J.-J. van de Beek, P. O. Borjesson, M.-L. Boucheret, D. Landstrom, J. M. Arenas, P. Odling, C. Ostberg, M. Wahlqvist, and S. K. Wilson, "A time and frequency synchronization scheme for multiuser OFDM," IEEE Journal of Selected Areas in Communications, vol. 17, no. 11, Apr 1999.

[10] Z. Wang, Y. Xin, and G. Mathew, "Iterative carrier-frequency offset estimation for generalized OFDMA uplink transmission," IEEE Transactions on Wireless Communications, vol. 8, no. 3, March 2009.

[11] Y. Yao and G. B. Giannakis, "Blind carrier frequency offset estimation in SISO, MIMO, and Multiuser OFDM systems," IEEE Transactions on Communications, vol. 53, no. 1, Jan 2005. 